\title{
Action Spectrum for Melatonin Regulation in Humans: Evidence for a Novel Circadian Photoreceptor
}

\author{
George C. Brainard, ${ }^{1}$ John P. Hanifin, ${ }^{1}$ Jeffrey M. Greeson, ${ }^{1}$ Brenda Byrne, ${ }^{1}$ Gena Glickman, ${ }^{1}$ \\ Edward Gerner, ${ }^{1}$ and Mark D. Rollag ${ }^{2}$ \\ ${ }^{1}$ Department of Neurology, Thomas Jefferson University, Philadelphia, Pennsylvania 19107, and ${ }^{2}$ Department of Anatomy, \\ Physiology and Genetics, Uniformed Services University of Health Sciences, Bethesda, Maryland 20814
}

\begin{abstract}
The photopigment in the human eye that transduces light for circadian and neuroendocrine regulation, is unknown. The aim of this study was to establish an action spectrum for lightinduced melatonin suppression that could help elucidate the ocular photoreceptor system for regulating the human pineal gland. Subjects (37 females, 35 males, mean age of $24.5 \pm 0.3$ years) were healthy and had normal color vision. Full-field, monochromatic light exposures took place between 2:00 and 3:30 A.M. while subjects' pupils were dilated. Blood samples collected before and after light exposures were quantified for melatonin. Each subject was tested with at least seven different irradiances of one wavelength with a minimum of 1 week between each nighttime exposure. Nighttime melatonin suppression tests ( $n=627$ ) were completed with wavelengths from 420 to $600 \mathrm{~nm}$. The data were fit to eight univariant, sigmoidal
\end{abstract}

fluence-response curves $\left(R^{2}=0.81-0.95\right)$. The action spectrum constructed from these data fit an opsin template $\left(R^{2}=\right.$ 0.91 ), which identifies $446-477 \mathrm{~nm}$ as the most potent wavelength region providing circadian input for regulating melatonin secretion. The results suggest that, in humans, a single photopigment may be primarily responsible for melatonin suppression, and its peak absorbance appears to be distinct from that of rod and cone cell photopigments for vision. The data also suggest that this new photopigment is retinaldehyde based. These findings suggest that there is a novel opsin photopigment in the human eye that mediates circadian photoreception.

Key words: melatonin; action spectrum; circadian; wavelength; light; pineal gland; neuroendocrine; photoreception; photopigment; human
Light is the primary stimulus for regulating circadian rhythms, seasonal cycles, and neuroendocrine responses in many species, including humans (Klein et al., 1991; Wehr, 1991). Furthermore, clinical studies have demonstrated that light therapy is effective for treating selected affective disorders, sleep problems, and circadian disruptions (Wetterberg, 1993; Lam, 1998). Currently, the ocular photoreceptors that transduce light stimuli for circadian regulation and the clinical benefits of light therapy are unknown.

The retinohypothalamic tract, a distinct neural pathway that mediates circadian regulation by light, projects from the retina to the suprachiasmatic nuclei (SCN) (Moore, 1983). A neural pathway extends from the SCN to the pineal gland (Klein et al., 1991; Morin, 1994). By this pathway, light and dark cycles are perceived through the mammalian eyes, entrain SCN neural activity, and, in turn, entrain the rhythmic secretion of melatonin from the pineal

\footnotetext{
Received March 22, 2001; revised May 17, 2001; accepted May 25, 2001.

This work was supported by National Institutes of Health Grant RO1NS36590 and NASA Cooperative Agreement NCC 9-58 with the National Space Biomedical Research Institute (to G.C.B.) and National Science Foundation Grant IBN9809916 and Department of Defense Grant R070HY (to M.D.R.). Input from many individuals was invaluable to this project. We gratefully acknowledge the support and technical assistance of Christine Alocillo, Jon Cooke, William Coyle, James Gardner, Frank Giunpa, Rick Guyer, Robert Glasgow, John McDevitt, John Monnier, Charles Nelson, Jeff Santman, and Donna Wittkowski. We also deeply appreciate the assistance from Laine Brainard, Dr. Ignacio Provencio, Dr. Britt Sanford, and Dr. William Thornton in assessing the data, developing graphs, and reviewing this manuscript. The inspiration for this work came from the 281 series of the Edgar Cayce readings.

Correspondence should be addressed to Dr. George C. Brainard, Department of Neurology, Thomas Jefferson University, 1025 Walnut Street, Suite 310, Philadelphia, PA 19107. E-mail: george.brainard@mail.tju.edu.

Copyright (C) 2001 Society for Neuroscience $\quad 0270-6474 / 01 / 216405-08 \$ 15.00 / 0$
}

gland. In virtually all species, melatonin secretion is high during the night and low during the day (Reiter, 1991; Arendt, 1998). In addition to entraining pineal rhythms, light exposure can acutely suppress melatonin secretion (Rollag and Niswender, 1976; Lewy et al., 1980). Acute, light-induced melatonin suppression is a broadly used indicator for photic input to the SCN, which has been used to elucidate the ocular and neural physiology for circadian regulation (Klein et al., 1991; Brainard et al., 1997).

Studies using rodents with retinal degeneration suggest that neither the rods nor cones used for vision participate in lightinduced melatonin suppression, circadian phase shifts, or photoperiodic responses (Pevet et al., 1984; Webb et al., 1985; Foster et al., 1991). Furthermore, enucleation of rodless, coneless transgenic mice abolishes light-induced circadian phase shifts and melatonin suppression (Lucas and Foster, 1999; Freedman et al., 1999). Similarly, light-induced melatonin suppression and circadian entrainment have been demonstrated in humans with complete visual blindness (Czeisler et al., 1995) and with specific color vision deficiencies (Ruberg et al., 1996). Together, these studies on different forms of visual blindness suggest that melatonin regulation is controlled, at least in part, by photoreceptors that differ from the known photoreceptors for vision.

A recent study has shown that monochromatic light at $505 \mathrm{~nm}$ is approximately four times stronger than $555 \mathrm{~nm}$ in suppressing melatonin in healthy humans (Brainard et al., 2001). Those results confirmed that the ocular photoreceptor primarily responsible for pineal melatonin regulation in humans is not the three cone system that mediates photopic vision. The new data reported here extend this work by forming an action spectrum from fluence-response curves at multiple visible wavelengths. 
Developing an action spectrum is a fundamental means for determining the input physiology for the circadian system. This photobiological technique has high utility for (1) defining the relative effectiveness of photons at different wavelengths for eliciting a biological response and (2) identifying the specific photopigment involved in that response (Lipson, 1994; Coohill, 1999). The specific aim of the present study was to characterize the wavelength sensitivity of the photoreceptor system responsible for providing circadian input to the human pineal gland by establishing an action spectrum for light-induced melatonin suppression. The experiments defined an action spectrum that fits a retinaldehyde opsin template and identified 446-477 $\mathrm{nm}$ as the most potent wavelength region for regulating melatonin. Univariance among the eight fluence-response curves suggests that a single photopigment is primarily responsible for melatonin suppression. These results suggest that there is a novel photopigment in the human eye that mediates circadian photoreception.

Preliminary reports of this work have been presented previously (Brainard et al., 1999b-e, 2000b).

\section{MATERIALS AND METHODS}

Study design. Action spectra are determined by comparing the number of photons required for the same biological effect at different wavelengths (Lipson, 1994; Coohill, 1999). The melatonin suppression action spectrum described here was formed from fluence-response curves at eight wavelengths between 440 and $600 \mathrm{~nm}$. A within-subjects design was used for each fluence-response curve. For each wavelength studied, a set of eight volunteers was exposed to a minimum of eight different light irradiances on separate nights with at least $6 \mathrm{~d}$ between exposures. At the completion of that work, it was determined that a probe of sensitivity to monochromatic light below $440 \mathrm{~nm}$ was needed. Consequently, a group of eight subjects was exposed to a single night of no light exposure and a single night of exposure to one irradiance of $420 \mathrm{~nm}$ light.

Subjects. Volunteers who were involved in shift work, planned longdistance jet travel before or during the study period, or had irregular sleeping schedules were excluded from this study. The subject drop-out rate was $7.9 \%$. The ethnic distribution of the 72 subjects who completed this study included 55 Caucasians, 9 Asians, 4 African Americans, 3 Hispanics, and 1 individual of unknown ethnicity. Subjects who had a relatively stable daily sleeping pattern, passed a physical exam for general and ocular health, and signed an approved Institutional Review Board consent document were accepted into this study. A total of 37 females and 35 males between 18 and 30 years old (mean \pm SEM age, $24.5 \pm 0.3$ ) completed the study. The self-reported mean \pm SEM weekday wake-up time among subjects was 7:06 A.M. $\pm 18 \mathrm{~min}$. All subjects were normal on the Ishihara and Farnsworth Munsell D-100 tests for color vision (mean \pm SEM Farnsworth Munsell score, $51.4 \pm 4.3$ ).

Light exposure protocol. Each experiment began at midnight when subjects entered a dimly lit room (10 lux or less). One drop of $0.5 \%$ cyclopentolate $\mathrm{HCl}$ was placed in each eye to dilate the subjects' pupils, and blindfolds were placed over their eyes. Subjects remained sitting upright for $120 \mathrm{~min}$ and listened to music on headphones or engaged in quiet conversation. While still blindfolded and just before 2:00 A.M., a 10 $\mathrm{ml}$ blood sample was taken by venipuncture of the antecubital vein. Subjects' blindfolds were then removed, and the subjects were exposed to the monochromatic light stimulus from 2:00 to 3:30 A.M. During light exposure, each subject's head rested in an ophthalmologic head holder facing a ganzfeld apparatus that provided a concave, patternless reflecting surface encompassing each subject's entire visual field (Fig. 1). During this 90 min exposure, subjects sat quietly, kept their eyes open, and gazed at a fixed target dot in the center of the ganzfeld dome. Subject compliance for keeping their eyes open and the subjects' pupil size were monitored by a miniature video camera inside the ganzfeld dome. If the subjects began to close their eyes during the exposure period, the experimenters reminded them to keep their eyes completely open. At 3:30 A.M., a second $10 \mathrm{ml}$ blood sample was taken by venipuncture, and the subjects were then permitted to leave the laboratory. Eight wavelengths were studied for this action spectrum $(440,460,480,505,530$, 555,575 , and $600 \mathrm{~nm}$ ). Across these wavelengths, each subject was exposed to complete darkness from 2:00 to 3:30 A.M. on their control night and to a set of irradiances covering a $4 \log$ unit photon density

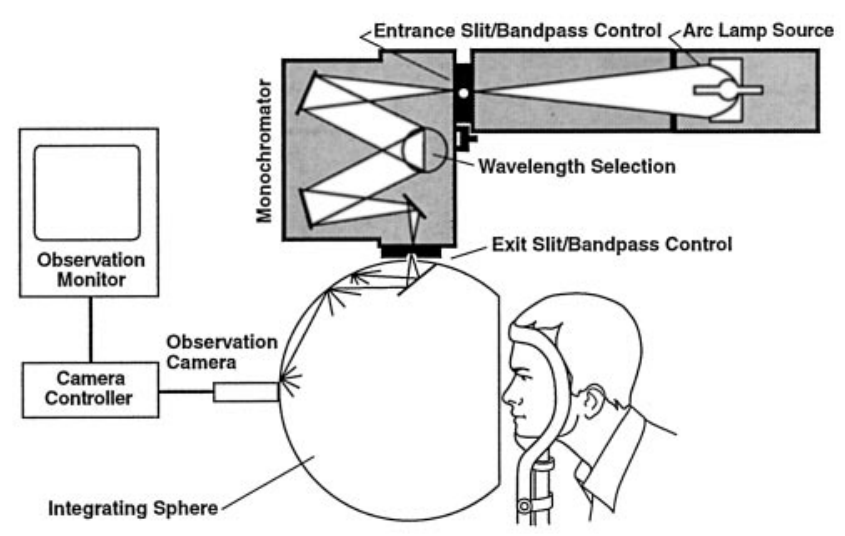

Figure 1. This diagram illustrates the experimental electronic, optic, and ganzfeld dome exposure array. This apparatus provides a uniform, patternless stimulus that encompasses the subject's entire visual field. For clarity, the subject's head is shown slightly withdrawn from the opening of the ganzfeld dome. During all light exposures, the subjects' bony orbits are completely enclosed in the dome walls, providing complete exposure of their visual fields.

range of $10^{10}$ to $10^{14}$ photons $/ \mathrm{cm}^{2}$ on exposure nights. For the probe of sensitivity to monochromatic light at $420 \mathrm{~nm}$, a group of eight subjects was exposed to a single night of no light exposure and a single night of exposure to $420 \mathrm{~nm}$ light at $31.8 \mu \mathrm{W} / \mathrm{cm}^{2}\left(5.58 \times 10^{13}\right.$ photons $\left./ \mathrm{cm}^{2}\right)$.

Light production and measurement. As shown in Figure 1, experimental light stimuli were produced by a 450 or $1200 \mathrm{~W}$ xenon arc lamp (Photon Technology Inc., Princeton, NJ). Each lamp was enclosed in a light-proof chamber and cooled by water circulation. An exit beam of light from each source was directed by a parabolic reflector, and, for the $1200 \mathrm{~W}$ lamps, excess heat in the light beam was reduced by a water filter. Monochromatic wavelengths (10-14.5 nm half-peak bandwidths) were produced by a grating monochromator, and light irradiance was controlled by a manual diaphragm. The resulting light beam was directed into the top area of a ganzfeld apparatus and reflected evenly off the walls of the ganzfeld dome into volunteers' eyes. The entire reflecting surface of the dome was coated with a white material (Spectralite) with a 95-99\% reflectance efficiency over the $400-760 \mathrm{~nm}$ range. Routine measurement of the light irradiance (in microwatts per square centimeter) was done with a Tektronix J16 Radiometer/Photometer with a J6512 irradiance probe (Tektronix, Beaverton, OR). Experimental light stimuli reflected from the ganzfeld dome were measured at volunteers' eye level immediately before and after the $90 \mathrm{~min}$ exposure. Additional measures were taken each $0.5 \mathrm{hr}$ of the exposure to ensure stimulus stability and enable readjustment of the intensity if it varied. These spot measures were taken with an $\mathrm{ft}-1^{\circ}$ meter (Minolta, Osaka, Japan). Spectroradiometric assessment of the monochromatic wavelengths at the level of subjects' corneas was done with a portable spectroradiometer with a fiber optic sensor (model S2000; Ocean Optics, Dunedin, FL). This equipment was calibrated with a standard lamp traceable to the National Institute of Standards and Technology.

In action spectroscopy, it is critical that the measured light stimuli are representative of the stimuli that actually reach the photoreceptors that mediate the photobiological response. In studies on light regulation of the circadian system, factors that can modify the measured stimulus before it reaches the photoreceptors include head and eye motion, squinting and eye closure, pupillary reflexes, and light transmission through the ocular media (Gaddy et al., 1993; Brainard et al., 1997). Most of these factors are controlled in the experimental technique described above. Concerning light transmission through ocular media, the cornea and aqueous and vitreous humors normally transmit nearly $100 \%$ of visible wavelengths to the retina and do not change substantively as the eyes age (Boettner and Wolter, 1962). In contrast, the aging human lens develops pigmentation that attenuates the transmission of shorter visible wavelengths to the retina (Lerman, 1987; Brainard et al., 1997). In the present study, restricting the age of volunteers to 18-30 years controlled this factor. Measurements of mean transmittance of 36 postmortem human lenses in this age range showed relatively even transmission from 440 to $600 \mathrm{~nm}$. In contrast, there was a mean $45 \%$ reduction in lens 
transmission at $420 \mathrm{~nm}$ compared with $460 \mathrm{~nm}$ (Brainard et al., 1997). Consequently, measured corneal light irradiances at $420 \mathrm{~nm}$ had to be adjusted to compensate for reduced stimulus transmission to the retina even in this relatively young study group.

Blood samples and melatonin assay. Blood samples were collected in glass vacutainers that contained EDTA. Plasma was separated by refrigerated centrif ugation, aliquoted into cryogenic vials, and stored at $-20^{\circ} \mathrm{C}$ until assay. Melatonin concentrations were assayed by radioimmunoassay using antiserum described by Rollag and Niswender (1976). Radiolabeled ligand was prepared by adding $10 \mu \mathrm{l}$ of a dioxane solution containing $1 \mu \mathrm{mol}$ of 5 -methoxytryptamine and $1 \mu \mathrm{mol}$ of tri- $N$-butylamine to $250 \mu \mathrm{Ci}(0.1 \mathrm{nmol})$ dried Bolton-Hunter Reagent (NEN, Boston, MA). The reaction was allowed to proceed for $1 \mathrm{hr}$ before adding $50 \mu \mathrm{l}$ of aqueous sucrose $(16 \mathrm{gm} / \mathrm{ml}$ electrophoresis buffer) and purifying product by disc gel electrophoresis. Duplicate aliquots of $200 \mu$ l of each unknown and control sample were extracted into $2 \mathrm{ml}$ of chloroform. The chloroform was removed in a SpeedVac centrifuge (Savant Instruments, Holbrook, NY) and resuspended in $200 \mu \mathrm{l}$ of assay buffer (PBS, pH 7.4, containing $0.1 \%$ gelatin with $100 \mathrm{mg} / \mathrm{l}$ thimerosal as a preservative). The extracts were washed twice with $3 \mathrm{ml}$ of petroleum ether and then evaporated to dryness in a SpeedVac before being resuspended in $200 \mu \mathrm{l}$ of deionized water. Approximately 50,000 cpm of radiolabeled ligand and a 1:256,000 dilution of antiserum (R1055; bleeding date of 9/16/74) was added to each unknown and a triplicate twofold geometric series of standards ranging in concentration from 0.201 to $200 \mathrm{pg}$ per $200 \mu \mathrm{l}$ of assay buffer. The final assay volume of buffer in each tube was $400 \mu \mathrm{l}$. At the end of the $48 \mathrm{hr}$ incubation period, $3 \mathrm{ml}$ of $95 \%$ ethanol $\left(4^{\circ} \mathrm{C}\right)$ was added to each assay tube, and the bound radioactivity was precipitated by centrifugation at $2000 \times g$ for $30 \mathrm{~min}$. The supernatant was decanted, and radioactivity in the precipitate was quantified. The quantity of melatonin immunoreactivity in the samples was calculated with the use of a computer program (M. L. Jaffe and Associates, Silver Spring, MD) (Davis et al., 1980). All solutions were maintained at $4^{\circ} \mathrm{C}$ throughout the radioimmunoassay procedure. Assay results were not corrected for recovery (which has proven to be $>95 \%$ in independent trials). The minimum detection limit of the assay is $0.5-2.0 \mathrm{pg} / \mathrm{ml}$.

Statistics. Two-tailed, paired Student's $t$ tests were used to assess statistical significance of raw melatonin change from 2:00 to 3:30 A.M. Percent melatonin change scores were determined by the following formula:

percent melatonin change score $=$

$$
100 \times \frac{03: 30 \mathrm{hr} \text { melatonin }-02: 00 \mathrm{hr} \text { melatonin }}{02: 00 \mathrm{hr} \text { melatonin }} .
$$

Percent melatonin change scores then were normalized to percent control-adjusted change scores by subtracting the control (no light) condition percent change scores for each subject from that same subject's light exposure score. This technique accounts for the normal individual rise or fall in plasma melatonin levels with respect to the light-induced changes (Gaddy et al., 1993; Brainard et al., 1997). For data from each wavelength, complete sets of preexposure melatonin values, percent melatonin change scores, and percent control-adjusted melatonin change scores were analyzed with one-way, repeated-measures ANOVA. Significant differences between groups were assessed with post hoc Scheffe $F$ tests with $\alpha$ set at 0.05 . The group of single fluence-response curves (one for each wavelength) was fitted to a parametric model in which the melatonin response $(Y)$ to a photon dose $(X)$ is predicted by the following: the theoretical initial $Y$-response $\left(0\right.$ dose) for the curve $\left(A_{1}\right)$; the theoretical final $Y$-response ("infinite" dose) for the curve $\left(\mathrm{A}_{2}\right)$; the dose producing a response halfway between $\mathrm{A}_{1}$ and $\mathrm{A}_{2}\left(\mathrm{X}_{50}\right.$ or $\left.\mathrm{ED}_{50}\right)$; and the slope estimator $(p)$ for the slope of the curve between $\mathrm{A}_{1}$ and $\mathrm{A}_{2}$. The equation is as follows:

$$
Y=\frac{A_{1}-A_{2}}{1+\left(X / X_{50}\right)^{p}}+A_{2}
$$

The computer program Origin 6.0 (Microcal, Northampton, MA) was used to fit the fluence-response curves to the data. From extensive experience in our laboratory, a saturating $90 \mathrm{~min}$ light exposure produces a maximum mean percent control-adjusted plasma melatonin suppression ranging from 60 to $80 \%$ depending on the particular group of subjects being tested (Gaddy et al., 1993; Ruberg et al., 1996; Wang et al., 1999; Brainard et al., 2000a, 2001). To form an analytical action spectrum, it is necessary to determine whether all fluence-response curves can be fit to a univariant sigmoidal curve (Coohill, 1991; Lipson, 1994, 1999). To do this, sigmoid curves were fitted to the five fluence-response curves between 440 and $530 \mathrm{~nm}$, which reached a mean percent controladjusted melatonin suppression of $60-80 \%$ by constraining the $A_{1}$ factor (theoretical initial $Y$-response) to 0 because no light exposure should yield a $0 \%$ control-adjusted plasma melatonin suppression. From this set of curves, a mean $\mathrm{A}_{2}$ (theoretical final $Y$-response or "infinite" dose for the curve) and a mean $p$ (slope estimator) was calculated. Subsequently, all eight data sets (including the data sets that did not reach saturation) were then fitted to sigmoid curves that constrained $\mathrm{A}_{2}$ and $p$ to these means and constrained $A_{1}$ to 0 . Each calculated curve was tested for goodness-of-fit of the data by coefficient of correlation.

Melatonin action spectrum. This action spectrum was formed from the photon density, which elicited the half-saturation constant $\left(\mathrm{ED}_{50}\right)$ of the percent control-adjusted melatonin suppression for each of the eight wavelengths. These half-saturation constants were derived from the eight univariant fluence-response curves described above. The half-saturation constants were then normalized to the maximum response and plotted as relative sensitivity. The relative quantum sensitivity from each group of subjects was then graphically plotted (quanta/wavelength) to illustrate the resultant action spectra for melatonin suppression in humans. A predicted peak sensitivity for this action spectrum was determined by fitting a vitamin $\mathrm{A}_{1}$-retinaldehyde photopigment template to the data by a modification of the method described by MacNichol et al. (1983). Specifically, the long wavelength limb of vitamin $\mathrm{A}_{1}$-based photopigments can be considered linear within the $10-90 \%$ sensitivity range when plotted on a frequency abscissa. To select the best fit vitamin $A_{1}$ template, the normalized $10-90 \%$ long wavelength melatonin $\mathrm{ED}_{50}$ data were fitted to a series of vitamin $A_{1}$-based templates within the $10-90 \%$ sensitivity range of the long-wavelength limbs of the templates (Partridge and De Grip, 1991). Pearson correlation coefficients derived from fitting the melatonin data to the templates indicated the optimum fitting template.

\section{RESULTS}

\section{Variations in pupillary dilation, exposure time, and melatonin assay}

Individuals vary slightly in their pupil size and response to mydriatic agents. Mean \pm SD pupillary dilation was $7.19 \pm 0.88 \mathrm{~mm}$ for all 72 subjects across all nights of exposures. There were no significant pupil size changes during the light exposures. Similarly, there is a small degree of variability in exact light exposure durations attributable to slight experimental delays. Across 627 single-subject experiments, the mean $\pm \mathrm{SD}$ exposure duration was $90.6 \pm 2.1 \mathrm{~min}$. A total of 53 assays were run to quantify melatonin in plasma samples collected during this project. Coefficients of variation calculated from control samples assayed as 19.2 and $90.0 \mathrm{pg} / \mathrm{ml}$ had 10.8 and $4.0 \%$ for intra-assay coefficients of variation, respectively. The inter-assay coefficients of variation were 13.5 and $10.2 \%$.

\section{Fluence-response data at $460 \mathrm{~nm}$}

Given that the predicted peak of the final action spectrum is 464 $\mathrm{nm}$, the full data complement, from raw melatonin values to a final fluence-response curve for the nearby monochromatic stimulus at $460 \mathrm{~nm}$, is illustrated in Figure 2. This fluence-response study at $460 \mathrm{~nm}$ was done with eight subjects (four males and four females). Across these subjects on all nights of testing, there were no significant differences $(F=0.70 ; p=0.69)$ between sets of preexposure values, indicating that baseline nocturnal melatonin levels were consistent across the different nights of study. The top graph in Figure 2 shows the mean + SEM preexposure and postexposure (2:00-3:30 A.M.) melatonin values (mean range, $72.1-29.3 \mathrm{pg} / \mathrm{ml}$ ). At $460 \mathrm{~nm}$, exposure to irradiances of 2.3 $\mu \mathrm{W} / \mathrm{cm}^{2}$ and lower did not significantly suppress plasma melatonin. In contrast, exposures of $3.1 \mu \mathrm{W} / \mathrm{cm}^{2}$ and higher elicited significant melatonin suppressions ( $p<0.03$ or less).

For comparative purposes, all melatonin data were converted 

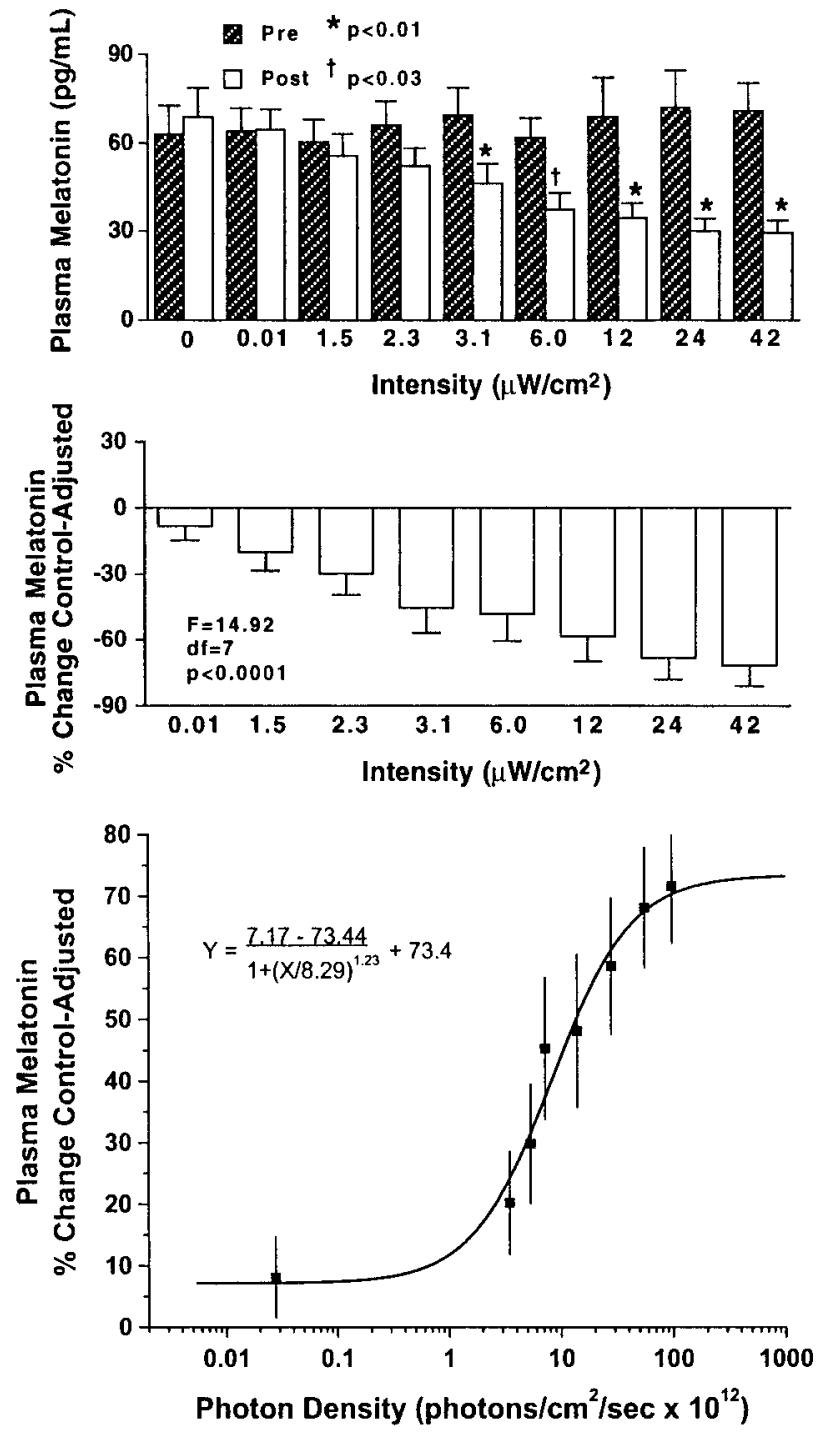

Figure 2. In the top two graphs, the bars represent group mean + SEM values of plasma melatonin relative to $460 \mathrm{~nm}$ monochromatic light exposure at different irradiances in one group of eight healthy subjects. The top shows plasma melatonin values before and after light exposure. There were no significant differences $(F=0.70 ; p=0.69)$ across preexposure mean melatonin values. Light irradiances at or above $3.1 \mu \mathrm{W} / \mathrm{cm}^{2}$ elicited significant melatonin suppression. The middle illustrates the subjects' plasma melatonin percent control-adjusted change scores. Progressively higher irradiance exposures at $460 \mathrm{~nm}$ produce progressively greater plasma melatonin percent control-adjusted change scores $(p<$ 0.0001). The bottom demonstrates the best-fit fluence-response curve for $460 \mathrm{~nm}$ exposures and percent control-adjusted melatonin suppression $\left(R^{2}=0.97\right)$. Each data point represents one group mean \pm SEM.

to plasma melatonin percent control-adjusted change scores. As illustrated in the middle graph of Figure 2, one-way, repeatedmeasures ANOVA showed a significant effect of light intensity on plasma melatonin percent control-adjusted change scores $(F=$ 14.92; $p<0.0001)$. Post hoc tests on plasma melatonin percent control-adjusted scores demonstrated that all intensities at or above $3.1 \mu \mathrm{W} / \mathrm{cm}^{2}$ significantly suppressed melatonin more than the $0.012 \mu \mathrm{W} / \mathrm{cm}^{2}$ stimulus $(p<0.05$ or less). Similarly, all irradiances at or above $12.1 \mu \mathrm{W} / \mathrm{cm}^{2}$ significantly suppressed melatonin more than the $1.5 \mu \mathrm{W} / \mathrm{cm}^{2}$ stimulus. Finally, both 24.2 and $42.2 \mu \mathrm{W} / \mathrm{cm}^{2}$ exposures elicited significantly higher plasma melatonin percentage of control-adjusted change scores compared with an irradiance of $2.3 \mu \mathrm{W} / \mathrm{cm}^{2}$.

The data from the middle graph of Figure 2 can be mathematically converted into a best-fit, sigmoidal curve that plots melatonin suppression against stimulus photon density. The specific formula for this curve is shown below and has a 0.97 coefficient of correlation $\left(R^{2}\right)$ :

$$
y=\frac{7.17-73.4}{1+(x / 8.29)^{1.23}}+73.4
$$

As shown in the bottom illustration in Figure 2, this curve illustrates the fluence-response interaction between mean \pm SEM melatonin percent control-adjusted change scores and the photon density of the monochromatic light.

\section{Fluence-response data for all eight wavelengths}

As shown in Figure 2, there is a clear, fluence-response relationship between graded photon densities of monochromatic $460 \mathrm{~nm}$ light and melatonin suppression. Data from each of the eight wavelengths tested in this study fit four-parameter sigmoidal curves with high coefficients of correlation. Specifically, wavelengths at 440, 460, 480, 505, 530, 555, 575, and $600 \mathrm{~nm}$ had respective coefficients of correlation $\left(R^{2}\right)$ : 0.99, 0.97, 0.95, 0.97, 0.98, 0.92, 0.96, and 0.97. As described in Materials and Methods, to form an analytical action spectrum, all fluence-response curves must be fit to a univariant sigmoidal curve (Lipson, 1994; Coohill, 1999). The univariant curve model for the data in this study has the factors of $\mathrm{A}_{1}=0, \mathrm{~A}_{2}=66.9$, and $p=1.27$. Figure 3 illustrates all eight univariant fluence-response curves from this study. As with previous circadian analytical action spectra (Takahashi et al., 1984; Provencio and Foster, 1995; Yoshimura and Ebihara, 1996), full-range fluence-response curves were not elicited above 550 nm. Despite this, standard photobiological curve-fitting methods could be used to fit the data from all eight wavelengths in the present study to univariant, sigmoidal functions. When fit to a univariant fluence-response curve with these factors, the data from exposures to 440, 460, 480, 505, 530, 555, 575, and $600 \mathrm{~nm}$ have high coefficients of correlation of $0.91,0.95,0.93,0.94,0.92$, $0.90,0.95$, and 0.81 , respectively.

\section{Melatonin suppression response to $420 \mathrm{~nm}$ at a single intensity}

Given the high sensitivity of subjects to short-wavelength light, as shown in Figure 4, it was determined that a probe of sensitivity to monochromatic light below $440 \mathrm{~nm}$ was needed. On the control night when the eight volunteers were exposed to darkness only, their raw mean melatonin levels at 2:00 and 3:30 A.M. were 69.4 and $76.0 \mathrm{pg} / \mathrm{ml}$, respectively. That small increase was not statistically significant $(t=-1.15 ; p=0.29)$. As shown in Figure 4, when these volunteers were exposed to $420 \mathrm{~nm}$ light at 31.8 $\mu \mathrm{W} / \mathrm{cm}^{2}\left(5.58 \times 10^{13}\right.$ photons $\left./ \mathrm{cm}^{2}\right)$, raw mean melatonin levels at 2:00 and 3:30 A.M. were 76.4 and $47.6 \mathrm{pg} / \mathrm{ml}$, respectively. That decrease in melatonin was statistically significant $(t=4.67 ; p<$ $0.003)$. For comparative purposes, this single melatonin suppression response was fitted to the univariant fluence-response curve formula used for all of the data in Figure 3. The resulting curve estimated a half-maximum $\left(\mathrm{X}_{50}\right.$ or $\left.\mathrm{ED}_{50}\right)$ melatonin suppression response for $420 \mathrm{~nm}$ of $1.83 \times 10^{13}$ photons $/ \mathrm{cm}^{2}$.

\section{Action spectrum for melatonin suppression}

Action spectra are determined by comparing the number of photons required for the same biological effect at different wave- 


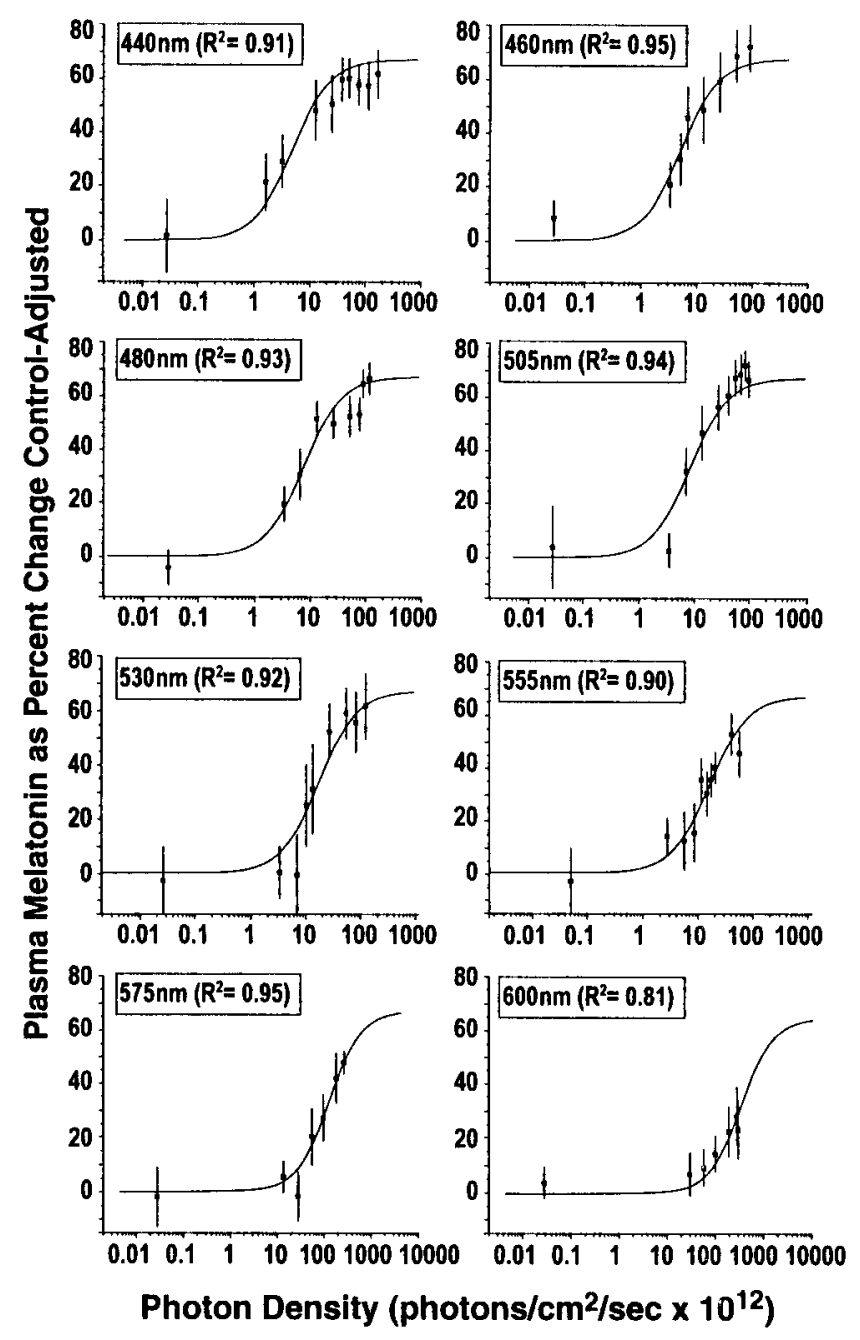

Figure 3. This figure illustrates the fitted univariant fluence-response curves for monochromatic light exposures and percent control-adjusted melatonin suppression for eight wavelengths of visible light. Each fluence-response curve is derived from eight healthy volunteers who participated in a complete, within-subjects experimental design. In each graph, the data points represent group means \pm SEM. Each curve has a high coefficient of correlation $(0.95-0.81)$.

lengths (Smith, 1989; Coohill, 1999). For this experiment, the action spectrum was formed from the photon density that elicited the half-saturation constant $\left(\mathrm{X}_{50}\right.$ or $\left.\mathrm{ED}_{50}\right)$ of the percent controladjusted melatonin suppression for each of the eight wavelengths. The half-saturation constants were derived from the eight univariant fluence-response curves shown in Figure 3 and the one estimated half-saturation constant from the data shown in Figure 4. The relative quantum sensitivity from each group of subjects was plotted in Figure 5 (quanta/wavelength) to illustrate the resultant action spectra for human melatonin suppression. When the data were aligned to the best-fit template for vitamin $A_{1}$ retinaldehyde photopigments, this action spectrum predicted a peak spectral sensitivity $\left(\lambda_{\max }\right)$ of $464 \mathrm{~nm}$. There was a strong coefficient of correlation between the data and this fitted opsin nomogram $\left(R^{2}=0.91\right)$.

\section{Comparison of action spectra}

The action spectrum for the photoreceptor system that provides input to the pineal gland appears to be distinct from the action spectra for the classical human visual photoreceptor systems. To

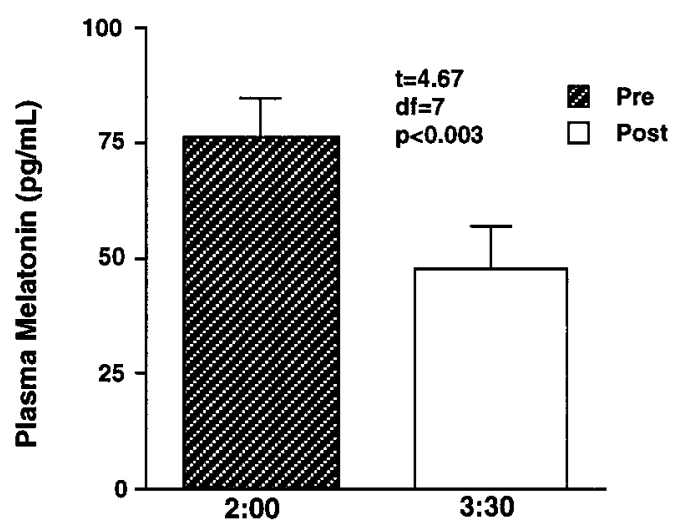

Figure 4. In this graph, the bars represent group mean \pm SEM plasma melatonin values before and after exposure to $31.8 \mu \mathrm{W} / \mathrm{cm}^{2}$ monochromatic light at $420 \mathrm{~nm}$ in eight healthy subjects. This light irradiance induced a significant melatonin suppression $(p<0.003)$.

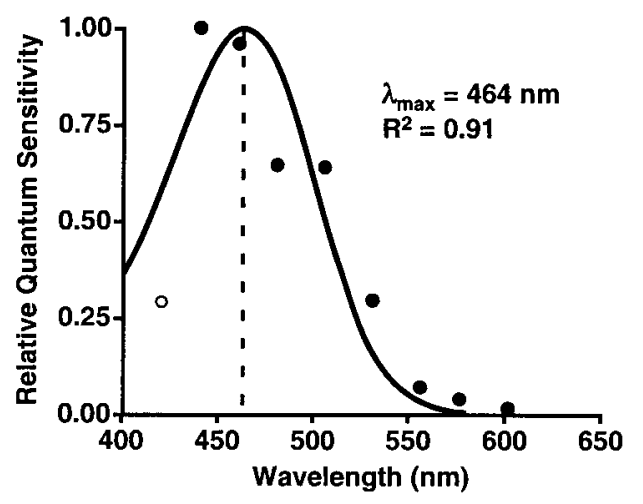

Figure 5. This graph demonstrates the action spectrum for percent control-adjusted melatonin suppression in 72 healthy human subjects. The filled circles represent the half-saturation constants of eight wavelengths from 440 to $600 \mathrm{~nm}$ that were normalized to the maximum response and plotted as log relative sensitivity. The open circle represents the estimated half-saturation constant derived from the $420 \mathrm{~nm}$ data. The solid curve portrays the best-fit template for vitamin $\mathrm{A}_{1}$ retinaldehyde photopigments, which predicts a maximal spectral absorbance $\left(\lambda_{\max }\right)$ of $464 \mathrm{~nm}$ (Partridge and De Grip, 1991). There is a high coefficient of correlation for fitting this opsin template to the melatonin suppression data $\left(R^{2}=0.91\right)$.

illustrate this, the maximal spectral absorbencies and long wavelength limbs of the human rod and cone photoreceptors that support vision (Stockman and Sharpe, 1999) are illustrated in Figure 6, along with the maximal spectral absorbance and long wavelength limb of the melatonin action spectrum. The shaded area around the melatonin action spectrum illustrates \pm SD for this function.

\section{DISCUSSION}

The action spectrum presented here is based on univariant fluence-response curves for melatonin suppression by eight monochromatic light wavelengths in healthy subjects. These data fit a vitamin $\mathrm{A}_{1}$ opsin template with 446-477 nm, providing the strongest circadian input for melatonin regulation. These results suggest that a novel photopigment in the human eye may be primarily responsible for melatonin regulation and may be distinct from the individual rod and cone photoreceptors for vision.

In developing a fluence-response curve, a complete withinsubjects experimental design produces the most reliable results. When subjects are studied over a 2-4 month period, however, 


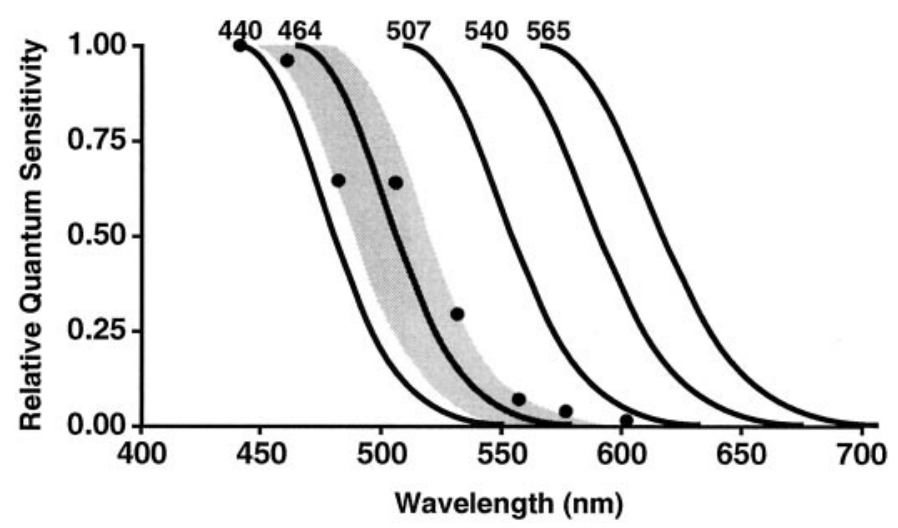

Figure 6. This figure illustrates a comparison of the melatonin suppression and visual action spectra. The maximal spectral response and long wavelength limb of the melatonin suppression template is plotted along with the maximal spectral response and long wavelength limbs of the human rods and cones that support vision (Stockman and Sharpe, 1999). The shaded area around the $464 \mathrm{~nm}$ template represents $\pm \mathrm{SD}$ from the data presented above.

lack of stability in the subjects' circadian entrainment can introduce variability in light-induced melatonin suppression. This study accepted only volunteers who reported regular bed and wake times, and their melatonin rhythms appeared to have been stable during the course of the study. As shown in the 2:00 A.M. melatonin values (Fig. 2, top), there were no significant differences between sets of preexposure values, indicating that baseline melatonin levels were consistent across the different study nights. This phenomenon has been documented for the $505 \mathrm{~nm}$ fluenceresponse group, as well as in other similarly controlled studies (Brainard et al., 1997, 2000a, 2001; Wang et al., 1998). This within-subject stability of the melatonin rhythm over time has been confirmed frequently in the literature (Waldhauser and Dietzel, 1985; Arendt, 1988, 1998).

The data from each of the eight wavelengths between 440 and $600 \mathrm{~nm}$ fit a univariant four-parameter sigmoidal curve with a high coefficient of correlation. The univariance of these curves is consistent with, but does not prove, the hypothesis that melatonin suppression is modulated by a single photoreceptor type. At this time, it is not certain that there is a univariant fluence-response function at $420 \mathrm{~nm}$ because only one intensity has been tested. It will be important to test for a full fluence-response curve at 420 $\mathrm{nm}$ to (1) clarify the precise sensitivity of the melatonin system to this wavelength and (2) determine if this wavelength is univariant with the fluence-response curves of the other eight wavelengths. Previous studies with animals and humans have illustrated similar fluence-response relationships for melatonin suppression and other circadian responses with monochromatic and broadspectrum light (Brainard et al., 1983, 1988; Podolin et al., 1987; McIntyre et al., 1989; Nelson and Takahashi, 1991; DkhissiBenyahya et al., 2000; Zeitzer et al., 2000). The initial attempts to define circadian and neuroendocrine responses to photons of different wavelengths began with polychromatic action spectra, which tested single irradiances of broader light bandwidths in various rodent species. These polychromatic action spectra were reasonably consistent in indicating that the spectral region between 450 and $550 \mathrm{~nm}$ provides the strongest stimulation of circadian and neuroendocrine responses in rodents (for review, see Brainard et al., 1999a). Analytic action spectra, based on sets of fluence-response curves at different monochromatic wave- lengths, are superior for identifying photoreceptors that mediate photobiological responses (Lipson, 1994; Coohill, 1999).

There are four analytic action spectra for circadian and neuroendocrine regulation in hamsters, rats, and mice (Takahashi et al., 1984; Bronstein et al., 1987; Provencio and Foster, 1995; Yoshimura and Ebihara, 1996). Data from these action spectra have been fitted to spectral sensitivity curves for retinal-based visual photopigments. This curve fitting is predicated on the assumption that a retinal-based molecule transduces light stimuli for circadian regulation and allows the prediction of the shape of the photopigment absorption spectrum, as well as its peak sensitivity $\left(\lambda_{\max }\right)$. Across these studies, which used different circadian endpoints, the predicted $\lambda_{\max }$ ranges from 480 to $511 \mathrm{~nm}$ and is surrounded by a broad region of high sensitivity. From these results, different photopigments have been suggested to be responsible for circadian regulation, including rhodopsin, a rhodopsin-like molecule, a middle wavelength cone photopigment, or an ultraviolet cone photopigment.

It is commonly believed that the photopic visual system has a peak wavelength sensitivity of $\sim 555 \mathrm{~nm}$ (Rodieck, 1998). Many investigators have hypothesized that the photopic visual system mediates circadian and neuroendocrine responses, because this part of the visual system is responsive to "bright" daytime levels of illumination. Previous data (Brainard et al., 2001) and those presented above do not support this hypothesis. The results clearly demonstrate that $555 \mathrm{~nm}$ is significantly weaker in suppressing melatonin compared with an equal photon density of 460 nm. Thus, the photopic system is not likely to be the primary input for circadian regulation. Demonstrating that the photopic visual system is not the principal phototransducer for melatonin regulation does not preclude it from having any role in circadian input. Indeed, recent studies suggest that visual cones may be involved in circadian regulation. Recordings from SCN neurons in rats indicate that the visual rods and cones provide input to cells of the rat SCN (Aggelopoulos and Meissl, 2000). Similarly, a human phase-shifting study suggests that, under some circumstances, the visual long wavelength-sensitive cone may also mediate circadian vision in humans (Zeitzer et al., 1997).

The data presented here do not support the hypothesis that any of the known visual photoreceptors provide the primary input for melatonin regulation. Figure 6 shows that none of the action spectra for individual visual photoreceptor systems match the action spectrum for melatonin suppression. If the photoreceptors that mediate vision in humans are not the primary photoreceptors for circadian regulation, what are the alternative candidates? Recent studies with various vertebrate species have identified several new molecules that may serve as circadian photopigments. These putative photopigments include both opsin-based molecules, such as vertebrate ancient opsin (Soni and Foster, 1997), melanopsin (Provencio et al., 1998), and peropsin (Sun et al., 1997), as well as non-opsin molecules, such as bilirubin (Oren, 1996) and cryptochrome (Miyamoto and Sancar, 1998). Among these new photopigments, only melanopsin has been specifically localized to the human neural retina (Provencio et al., 2000), and cryptochrome has been localized to the mouse neural retina (Miyamoto and Sancar, 1998). Cryptochromes have been studied extensively as circadian photoreceptors in plants and insects (Ahmad and Cashmore, 1993; Stanewsky et al., 1998) and have been proposed as circadian photoreceptors in mammals (Miyamoto and Sancar, 1998; Thresher et al., 1998). The contention that cryptochromes serve as circadian photoreceptors in humans 
or other mammals, however, remains controversial (Griffin et al., 1999; van der Horst et al., 1999; von Schantz et al., 2000).

The action spectrum presented here matches a vitamin $\mathrm{A}_{1}$ retinaldehyde photopigment template that supports the hypothesis that one of the new opsin photopigment candidates provides primary photic input for melatonin regulation in humans. The molecular identification of candidate opsin or non-opsin photoreceptors and their localization in the retina and/or neural components of the circadian system make them well suited to act as circadian phototransducers. However, functional data confirming any of these molecules as having a direct role in mammalian circadian photoreception is currently lacking. Furthermore, caution should be exercised in generalizing results from plants, insects, fish, amphibians, and rodents to humans.

Are the effects of light on melatonin suppression relevant to general circadian regulation? Studies have shown that hamsters have a higher intensity threshold for light-induced phase-shifts of wheel-running rhythms than for melatonin suppression (Nelson and Takahashi, 1991). Recently, however, a study on humans showed that the $50 \%$ response sensitivity for circadian phase shifting (119 lux) was only slightly higher than that for melatonin suppression (106 lux) with white light (Zeitzer et al., 2000). It is possible that there are separate photoreceptors for mediating circadian entrainment versus acute suppression of melatonin. It is reasonable, however, to hypothesize that a variety of nonvisual effects of light, such as melatonin suppression, entrainment of circadian rhythms, and possibly some clinical responses to light, are mediated by a shared photoreceptor system. Additional experiments are needed to test this hypothesis.

In general, relatively high light illuminances ranging from 2500 to 12,000 lux are used for treating winter depression, selected sleep disorders, and circadian disruption (Wetterberg, 1993; Lam, 1998). Although these light levels are therapeutically effective, some patients complain that they produce side effects of visual glare, visual fatigue, photophobia, ocular discomfort, and headache. Determining the action spectrum for circadian regulation may lead to improvements in light therapy. Total illuminances for treating a given disorder can be reduced as the wavelength emissions of the therapeutic equipment are optimized.

Modern industrialized societies use light extensively in homes, schools, work places, and public facilities to support visual performance, visual comfort, and aesthetic appreciation within the environment. Given that light is also a powerful regulator of the human circadian system, future lighting strategies will need to provide illumination for human visual responses, as well as homeostatic responses. The action spectrum presented here suggests that there are separate photoreceptors for visual and circadian responses to light in humans. Hence, new approaches to architectural lighting may be needed to optimally stimulate both the visual and circadian systems.

In conclusion, this study characterizes the wavelength sensitivity of the ocular photoreceptor system for regulating the human pineal gland by establishing an action spectrum for light-induced melatonin suppression. The results identify the 446-477 nm portion of the spectrum as the most potent wavelengths providing circadian input for regulating melatonin secretion. These data suggest that the primary photoreceptor system for melatonin suppression is distinct from the rod and cone photoreceptors for vision. Finally, this action spectrum suggests that there is a novel retinaldehyde photopigment that mediates human circadian photoreception. These findings open the door for optimizing the use of light in both therapeutic and architectural applications.

\section{REFERENCES}

Aggelopoulos NC, Meissl H (2000) Responses of neurones of the rat suprachiasmatic nucleus to retinal illumination under photopic and scotopic conditions. J Physiol (Lond) 523:211-222.

Ahmad M, Cashmore AR (1993) HY4 gene of $A$. thaliana encodes a protein with characteristics of a blue-light photoreceptor. Nature 366:162-166.

Arendt J (1988) Melatonin [review]. Clin Endocrinol 29:205-229.

Arendt J (1998) Melatonin and the pineal gland: influence on mammalian seasonal and circadian physiology. Rev Reprod 3:13-22.

Boettner EA, Wolter JR (1962) Transmission of the ocular media. Invest Ophthalmol Vis Sci 1:776-783.

Brainard GC, Richardson BA, King TS, Matthews SA, Reiter RJ (1983) The suppression of pineal melatonin content and $N$-acetyltransferase activity by different light irradiances in the Syrian hamster: a doseresponse relationship. Endocrinology 113:293-296.

Brainard GC, Lewy AJ, Menaker M, Miller LS, Fredrickson RH, Weleber RG, Cassone V, Hudson D (1988) Dose-response relationship between light irradiance and the suppression of melatonin in human volunteers. Brain Res 454:212-218.

Brainard GC, Rollag MD, Hanifin JP (1997) Photic regulation of melatonin in humans: ocular and neural signal transduction. J Biol Rhythms $12: 537-546$

Brainard GC, Greeson JM, Hanifin JP (1999a) Action spectra for circadian and neuroendocrine regulation in mammals. In: Measurements of optical radiation hazards (Matthes R, Sliney D, DiDomenico S, Murray P, Phillips R, Wengraitis S, eds), pp 131-142. Munich: International Commission on Non-Ionizing Radiation Protection.

Brainard G, Greeson J, Hanifin J, Byrne B, Gerner E, Rollag M (1999b) Development of an action spectrum for melatonin suppression in humans. Photochem Photobiol 69:6S.

Brainard G, Rollag M, Hanifin J, Greeson J, Byrne B, Gerner E (1999c) Action spectrum for melatonin regulation in humans. Presented at 8 th European Pineal Society Colloqium, Tours, France, July 3-7.

Brainard G, Rollag M, Hanifin J, Greeson J, Byrne B, Gerner E (1999d) Circadian photoreception: action spectrum for melatonin regulation in humans. Soc Neurosci Abstr 25:2064.

Brainard GC, Byrne B, Hanifin JP, Greeson JM, Gerner E, Rollag MD (1999e) Development of an action spectrum for melatonin regulation: potential relevance to light therapy. Soc Light Treat Biol Rhythms Abstr 11:1.

Brainard GC, Rollag MD, Hanifin JP, van den Beld G, Sanford B (2000a) The effect of polarized versus non-polarized light on melatonin regulation in humans. Photochem Photobiol 71:766-770.

Brainard G, Rollag M, Hanifin J, Greeson J, Byrne B, Glickman G, Gardner J, Sanford B, Gerner E' (2000b) Circadian photoreception in humans: action spectrum for melatonin suppression. Presented at 7 th Meeting of the Society for Research on Biological Rhythms, Jacksonville, FL, May 10-13.

Brainard GC, Hanifin JP, Rollag MD, Greeson J, Byrne B, Glickman G, Gerner E, Sanford B (2001) Human melatonin regulation is not mediated by the three cone photopic visual system. J Clin Endocrinol Metab 86:433-436.

Bronstein DM, Jacobs GH, Haak KA, Neitz J, Lytle LD (1987) Action spectrum of the retinal mechanism mediating nocturnal light-induced suppression of rat pineal gland $N$-acetyltransferase. Brain Res 406:352-356.

Coohill TP (1991) Action spectra again? Photochem Photobiol 54:859-870.

Coohill TP (1999) Photobiological action spectra: what do they mean? In: Measurements of optical radiation hazards (Matthes R, Sliney D, DiDomenico S, Murray P, Phillips R, Wengraitis S, eds), pp 27-39. Munich: International Commission on Non-Ionizing Radiation Protection.

Czeisler CA, Shanahan TL, Klerman EB, Martens H, Brotman DJ, Emens JS, Klein T, Rizzo III JF (1995) Suppression of melatonin secretion in some blind patients by exposure to bright light. N Engl J Med 332:6-11.

Davis SE, Munson PJ, Jaffe ML, Rodbard D (1980) Radioimmunoassay data processing with a small programmable calculator. J Immunoassay 1:15-25.

Dkhissi-Benyahya O, Sicard B, Cooper HM (2000) Effects of irradiance and stimulus duration on early gene expression (fos) in the suprachiasmatic nucleus: temporal summation and reciprocity. J Neurosci 20:7790-7797.

Foster RG, Provencio I, Hudson D, Fiske S, DeGrip W, Menaker M (1991) Circadian photoreception in the retinally degenerate mouse $(r d / r d)$. J Comp Physiol [A] 169:39-50.

Freedman MS, Lucas RJ, Soni B, von Schantz M, Muñoz M, David-Gray Z, Foster RG (1999) Regulation of mammalian circadian behavior by non-rod, non-cone, ocular photoreceptors. Science 284:502-504.

Gaddy JR, Rollag MD, Brainard GC (1993) Pupil size regulation of threshold of light-induced melatonin suppression. J Clin Endocrinol Metab 77:1398-1401. 
Griffin EA, Staknis D, Weitz CJ (1999) Light-independent role of Cry1 and Cry2 in the mammalian circadian clock. Science 286:768-771.

Klein DC, Moore RY, Reppert SM (1991) Suprachiasmatic nucleus: the mind's clock. Oxford: Oxford UP.

Lam RW (1998) Seasonal affective disorder and beyond: light treatment for SAD and non-SAD disorders. Washington, DC: American Psychiatric Press.

Lerman S (1987) Chemical and physical properties of the normal and aging lens: spectroscopic (UV, fluorescence, phosphorescence, and NMR) analyses. Am J Opt Physiol Optics 64:11-22.

Lewy AJ, Wehr TA, Goodwin FK, Newsome DA, Markey SP (1980) Light suppresses melatonin secretion in humans. Science 210:1267-1269.

Lipson ED (1994) Action spectroscopy: methodology. In: Organic photochemistry and photobiology (Horspool WM, Song P-S, eds), pp 1257-1266. New York: CRC.

Lucas RJ, Foster RG (1999) Neither functional rod photoreceptors nor rod or cone outer segments are required for the photic inhibition of pineal melatonin. Endocrinology 140:1520-1524.

MacNichol EF, Levine JS, Mansfield RJW, Lipetz LE, Collins BA (1983) Microspectrophotometry of visual pigments in primate photoreceptors. In: Colour vision (Mollon JD, Sharpe LT, eds), pp 14-38. Cambridge, UK: Academic.

McIntyre IM, Norman TR, Burrows GD, Armstrong SM (1989) Human melatonin suppression by light is intensity dependent. J Pineal Res 6:149-156.

Miyamoto Y, Sancar A (1998) Vitamin B2-based blue-light photoreceptors in the retinohypothalamic tract as the photoactive pigments for setting the circadian clock in mammals. Proc Natl Acad Sci USA 95:6097-6102.

Moore RY (1983) Organization and function of a central nervous system circadian oscillator: the suprachiasmatic hypothalamic nucleus. Fed Proc 42:2783-2789.

Morin LP (1994) The circadian visual system. Brain Res Brain Res Rev 19:102-127.

Nelson DE, Takahashi JS (1991) Comparison of visual sensitivity for suppression of pineal melatonin and circadian phase-shifting in the golden hamster. Brain Res 554:272-277.

Oren DA (1996) Humoral phototransduction: blood is a messenger. The Neuroscientist 2:207-210.

Partridge JC, De Grip WJ (1991) A new template for rhodopsin (vitamin A1 based) visual pigments. Vision Res 31:619-630.

Pevet P, Heth G, Hiam A, Nevo E (1984) Photoperiod perception in the blind mole rat (Spalax ehrenbergi, Nehring): involvement of the Harderian gland, atrophied eyes, and melatonin. J Exp Zool 232:41-50.

Podolin PC, Rollag MD, Brainard GC (1987) The suppression of nocturnal pineal melatonin in the Syrian hamster: dose-response curves at $500 \mathrm{~nm}$ and $360 \mathrm{~nm}$. Endocrinology 121:266-270.

Provencio I, Foster RG (1995) Circadian rhythms in mice can be regulated by photoreceptors with cone-like characteristics. Brain Res 694:183-190.

Provencio I, Jiang G, De Grip WJ, Hayes WP, Rollag MD (1998) Melanopsin: an opsin in melanophores, brain, and eye. Proc Natl Acad Sci USA 95:340-345.

Provencio I, Rodriguez IR, Jiang G, Hayes WP, Moreira EF, Rollag MD (2000) A novel human opsin in the inner retina. J Neurosci 20:600-605.

Reiter RJ (1991) Pineal melatonin: cell biology of its synthesis and of its physiological interactions. Endocr Rev 12:151-180.

Rodieck RW (1998) The first steps in seeing. Sunderland, MA: Sinauer.
Rollag MD, Niswender GD (1976) Radioimmunoassay of serum concentrations of melatonin in sheep exposed to different lighting regimens. Endocrinology 98:482-489.

Ruberg FL, Skene DJ, Hanifin JP, Rollag MD, English J, Arendt J, Brainard GC (1996) Melatonin regulation in humans with color vision deficiencies. J Clin Endocrinol Metab 81:2980-2985.

Smith KC (1989) The science of photobiology. New York: Plenum.

Soni BG, Foster RG (1997) A novel and ancient vertebrate opsin. FEBS Lett 406:279-283.

Stanewsky R, Kaneko M, Emery P, Beretta B, Wager-Smith K, Kay SA, Rosbash M, Hall JC (1998) The $c r y^{b}$ mutation identifies cryptochrome as a circadian photoreceptor in Drosophila. Cell 95:681-692.

Stockman A, Sharpe LT (1999) Cone spectral sensitivities and color matching. In: Color vision: from genes to perception (Gegenfurtner KR, Sharpe LT, eds), pp 53-87. Cambridge, UK: Cambridge UP.

Sun H, Gilbert DJ, Copeland NG, Jenkins NA, Nathans J (1997) Peropsin, a novel visual pigment-like protein located in the apical microvilli of the retinal pigment epithelium. Proc Natl Acad Sci USA 94:9893-9898.

Takahashi JS, DeCoursey PJ, Bauman L, Menaker M (1984) Spectral sensitivity of a novel photoreceptive system mediating entrainment of mammalian circadian rhythms. Nature 308:186-188.

Thresher RJ, Vitaterna MH, Miyamoto Y, Kazantsev A, Hsu DS, Petit C, Selby CP, Dawut L, Smithies O, Takahashi JS, Sancar A (1998) Role of mouse cryptochrome blue-light photoreceptor in circadian photoresponses. Science 282:1490-1494.

van der Horst GTJ, Muijtjens M, Kobayashi K, Takano R, Kanno S, Takao M, de Wit J, Verkerk A, Eker APM, van Leenen D, Buijs R, Bootsma D, Hoeijmakers JHJ, Yasui A (1999) Mammalian Cry1 and Cry2 are essential for maintenance of circadian rhythms. Nature 398:627-630.

von Schantz M, Provencio I, Foster RG (2000) Recent developments in circadian photoreception: more than meets the eye. Invest Ophthalmol Vis Sci 41:1605-1607.

Waldhauser F, Dietzel M (1985) Daily and annual rhythms in human melatonin secretion: role in puberty control. Ann NY Acad Sci 453:205-214.

Wang JY, Hanifin JP, Rollag MD, Brainard GC (1999) Ocular regulation of the human pineal gland: the significance of total retinal exposure for melatonin suppression. In: Biologic effects of light 1998 (Holick M, Jung EG, eds), pp 367-374. Boston: Kluwer Academic.

Webb SM, Champney TH, Lewinski AK, Reiter RJ (1985) Photoreceptor damage and eye pigmentation: influence on the sensitivity of rat pineal $N$-acetyltransferase activity and melatonin levels to light at night. Neuroendocrinology 40:205-209.

Wehr TA (1991) The durations of human melatonin secretion and sleep respond to changes in daylength (photoperiod). J Clin Endocrinol Metab 73:1276-1280.

Wetterberg L (1993) Light and biological rhythms in man. Stockholm: Pergamon.

Yoshimura T, Ebihara S (1996) Spectral sensitivity of photoreceptors mediating phase-shifts of circadian rhythms in retinally degenerate $\mathrm{CBA} / \mathrm{J}(\mathrm{rd} / \mathrm{rd})$ and normal CBA/N $(+/+)$ mice. J Comp Physiol [A] 178:797-802.

Zeitzer JM, Kronauer RE, Czeisler CA (1997) Photopic transduction implicated in human circadian entrainment. Neurosci Lett 232:135-138.

Zeitzer JM, Dijk D-J, Kronauer RE, Brown EN, Czeisler CA (2000) Sensitivity of the human circadian pacemaker to nocturnal light: melatonin phase resetting and suppression. J Physiol (Lond) 526:695-702. 